\title{
VIOLÊNCIA E VULNERABILIDADE: UM PANORAMA DA PRODUÇÃO CIENTÍFICA
}

\author{
Maria de Lourdes Denardin Budó* \\ Silvana de Oliveira Silva** \\ Maria Denise Schimith** \\ Mariane Rossato** \\ Adonias Santos da Rosa** \\ Letícia Pieniz Zimmermann** \\ Clarissa Potter** \\ Pâmela Almeida Batista** \\ Silvana Cruz da Silva**
}

\section{RESUMO}

Objetivo: O estudo exploratório, descritivo e bibliográfico objetivou investigar qual a produção do conhecimento científico sobre o tema violência e vulnerabilidade tendo em vista ser um assunto ainda pouco explorado na área da saúde. Método: Foi realizada uma pesquisa sistematizada nas bases de dados da BIREME no mês de junho do ano de dois mil e nove, com os descritores violência e vulnerabilidade nos últimos dez anos, sendo utilizado o método integrado. Resultado: Após a seleção, restaram quinze artigos os quais foram lidos na íntegra e posteriormente analisados quanto aos aspectos quantitativos e qualitativos. Desse estudo, emergiram duas categorias: estado e contexto social como geradores de violência e vulnerabilidade; e implicações da violência e vulnerabilidade nos serviços de saúde. Conclusão: A pesquisa revelou que ainda existem poucas produções acerca da temática em questão, diante de sua relevância e repercussões para a sociedade, sendo necessário ampliar os conhecimentos por meio de estudos de cunho qualitativos.

Descritores: Enfermagem; Violência; Vulnerabilidade.

\section{VIOLENCES AND VULNERABILITY: A CIENTIFIC PRODUCTION'S PANORAM}

\begin{abstract}
Objective: The exploratory, descriptive and bibliographic study, purposed to investigate what is the scientific knowledge production about the violence and vulnerability thematic, because it has been a target of studies that is still not much explored in the health area. Method: It was realized a systematized research at the dado's base of BIREME on the month of June of the year two thousand and nine, with the describer of violence and vulnerability in the last ten years, was used the integrated method. Result: After the selection was left fifteen articles which were read in its totality and afterward analyzed its qualitative and quantitative aspects. From this study emerged two categories: State and Social Context as generators of violence, vulnerability; violence implications and health service vulnerability. Conclusion: The research revealed that still exist few productions about the thematic in question, before its relevance and repercussion to the society, being necessary to amplify the knowledge's by studies with a qualitative matrix.
\end{abstract}

Describers: Nursing; Violence; Vulnerability.

\section{VIOLENCIAY YULNERABILIDAD: UN PANORAMA DE LOS CIENTÍFICOS}

\section{RESUMEN}

Objetivo: El estudio exploratorio, descriptivo y bibliográfico objetivó investigar respecto a la producción del conocimiento científico sobre el tema: violencia y vulnerabilidad, considerando que es un asunto aún poco explorado en el área de la salud. Método: fue realizada una pesquisa sistematizada basada en los datos de la BIREME en el mes de junio, del año 2009, con los descriptores violencia y vulnerabilidad, en los últimos dez años, siendo utilizado el método integrado. Resultado: tras la selección, restaran quince artículos que fueran leídos en su totalidad y, posteriormente, analizados cuanto a los aspectos cuantitativos y cualitativos. De ese estudio emergieran dos categorías: estado y contexto social como generadoras de la violencia y vulnerabilidad en los servicios de la salud. Conclusión: la investigación reveló que todavía existen pocas producciones acerca de la temática en cuestión, delante de su relevancia y repercusión para la

\footnotetext{
* Enfermeira, docente do Departamento de Enfermagem da UFSM. Área de atuação: Saúde coletiva; Saúde do adulto, família e envelhecimento; Cuidado cultural.

** Universidade Federal de Santa Maria
} 
sociedad, siendo necesario ampliar los conocimientos por intermedio de estudios de fondo cualitativos.

Descriptores: Enfermería; Violencia; Vulnerabilidad.

\section{INTRODUÇÃO}

Nos últimos anos, a temática violência e vulnerabilidade tem sido alvo de estudos na área da saúde tanto pelo impacto que provoca na qualidade de vida, pelas lesões físicas, psíquicas e morais que acarreta, como pelas exigências de atenção e cuidados nos serviços de saúde. ${ }^{1}$

Estudos revelam um crescente aumento da morbimortalidade oriunda da violência. No Brasil, as mortes por causas externas ocupam o segundo lugar no perfil da mortalidade geral e é a primeira causa de óbitos na faixa etária de 5 a 49 anos. $^{2}$ Desse modo, deve-se encarar a violência como um objeto da intersetorialidade, pois, o perfil de mortalidade e da morbidade da população brasileira hoje é marcado mais pelas condições, situações e estilos de vida do que pelas enfermidades tradicionais. ${ }^{1}$

Assim, torna-se necessário compreender qual a concepção de violência da qual se fala. Nesse estudo, compreende-se a violência como as "ações humanas de indivíduos, grupos, classes, nações que ocasionam a morte de outros seres humanos ou que afetam sua integridade fisica, moral, mental ou espiritual". $2: 2$ Nessa concepção "só se pode falar em violências, pois se trata de uma realidade plural, diferenciada e cujas especificidades precisam ser conhecidas" 2:2

Diante do exposto, torna-se imprescindível analisar a violência na perspectiva da vulnerabilidade, pois a interface que ocorre entre esses conceitos expande o leque de ações possíveis. Sabe-se que o termo vulnerabilidade alcançou destaque no campo da AIDS pelo fato de perceber que a epidemia respondia a determinantes cujos alcances iam além da unicausalidade. Esta percepção ampliada é aplicável a qualquer dano ou condição de interesse para a saúde pública, porque identifica as razões do evento tanto à suscetibilidade orgânica quanto à forma de estruturação de programas de saúde, passando por aspectos comportamentais, culturais, econômicos e políticos. ${ }^{3}$ Para tanto, pode-se conceituar vulnerabilidade como o
[...] movimento de considerar a chance de exposição das pessoas ao adoecimento como a resultante de um conjunto de aspectos não apenas individuais, mas também coletivos, contextuais, que acarretam maior suscetibilidade à infecção e ao adoecimento e, de modo inseparável, maior ou menor disponibilidade de recursos de todas as ordens para se proteger de ambos. 3:123

Desta maneira, esse estudo é fruto do trabalho de estudantes e docentes de graduação e pós-graduação em enfermagem do Grupo de Pesquisa Cuidado Saúde e Enfermagem que desenvolve pesquisas relacionando essa temática com a área da saúde coletiva.

A partir desses conceitos fica evidente a ligação dos mesmos com o cuidado em saúde. Nesse sentido destaca-se a importância dessa investigação, tendo em vista ser um tema ainda pouco explorado na área da saúde. Diante disso, esse estudo é fruto do trabalho de estudantes e docentes de graduação e pós-graduação em enfermagem do Grupo de Pesquisa Cuidado, Saúde e Enfermagem que desenvolve pesquisas relacionando essa temática na área da saúde coletiva. Para tanto, objetivou-se investigar a produção do conhecimento acerca da violência e vulnerabilidade, a fim de contribuir na instrumentalização e atualização de estudantes, docentes e trabalhadores da saúde na temática.

\section{MATERIAIS E MÉTODOS}

Realizou-se um estudo do tipo exploratório descritivo com busca no banco de dados da Biblioteca Virtual de saúde da BIREME, no mês março de 2010. Primeiramente, fez-se a consulta nos Descritores em Ciências da Saúde (DECS), a fim de determinar os descritores adequados para a temática, sendo os escolhidos, Violência e Vulnerabilidade. A pesquisa foi realizada pelo método integrado obtendo-se 75 publicações, destas, 47 artigos, 16 teses e oito monografias.

A seleção das produções científicas desenvolveuse a partir da leitura dos títulos e dos resumos. Utilizaram-se como critérios de seleção: a inclusão de 
artigos, textos completos, idiomas em português, espanhol e inglês, ano de publicação de 2000 a 2010, proporcionando a discussão na perspectiva de dez anos. Foram excluídos os trabalhos sem resumo, que não estivessem disponíveis na íntegra ou não se referirem especificamente ao tema.

Assim, da pesquisa sistematizada nas bases de dados da BIREME, restaram para análise quinze produções científicas, sendo sete estudos presentes nas duas bases LILACS (Literatura Latino-Americana e do Caribe em Ciências) e SCIELO (Scientific Electronic Library Online); duas na SCIELO e na Saúde na Adolescência (ADOLESC), uma produção na ADOLESC e quatro na LILACS e uma produção somente na SCIELO.

A análise dos artigos seguiu os passos propostos por Minayo (2008) para a análise temática. Para tanto, foi realizada a leitura flutuante e exaustiva dos estudos, a construção do corpus, tratamento dos dados e interpretação. Dessa forma, emergiram as categorias: estado e contexto social geradores da violência e vulnerabilidade; implicações da violência e vulnerabilidade nos serviços de saúde.

\section{RESULTADOS}

De acordo com o ano de publicação, dos quinze artigos analisados, 2006 foi o mais relevante, apresentando cinco publicações, seguido de 2007 com três, 2008 com dois artigos e os anos de 2000, 2002, 2003, 2005 e 2009 com apenas uma publicação cada.

Percebe-se que, dentre os estudos nacionais e publicados em revistas da América Latina, o Brasil é destaque na produção científica sobre a temática, com treze artigos publicados, sendo que destes, dois foram publicados em inglês. Os demais artigos foram publicados pelo Chile e pela Argentina, com uma produção cada. $\mathrm{Na}$ análise da produção no Brasil, verificou-se que o estado de São Paulo aparece com o maior número, seis publicações, seguido da Bahia com três publicações, Rio Grande do Sul com três pesquisas e o Rio de Janeiro apresenta uma publicação.

Destacam-se oito artigos resultantes de pesquisa, sendo sete de pesquisa quantitativa e uma de qualitativa. Os demais foram quatro publicações sobre reflexão teórica e três relatos de experiência. Este resultado reflete a intenção dos estudiosos, ao realizar estudos quantitativos, de revelar dados, indicadores e tendências observáveis. ${ }^{4}$ No entanto, acredita-se serem imprescindíveis também pesquisas de cunho qualitativo para esta temática, tendo em vista que se aplica ao estudo da história, das relações, das representações, das crenças, das percepções e das opiniões, produtos das interpretações que os seres humanos fazem a respeito de como vivem, constroem seus artefatos e a si mesmos, sentem e pensam. ${ }^{4}$

Obtiveram-se produções de diversos periódicos, dos quais se pode citar: três artigos publicados na revista Saúde e Sociedade e dois na Revista Latino Americana de Enfermagem, dois no Caderno Saúde Pública. O restante dos artigos foram publicados em número de um nos seguintes periódicos: Psicologia e Sociologia; Ciências e Saúde Coletiva; Saúde Pública em Perspectiva; Ciência e Enfermeria; Estudo e Pesquisa em Psicologia; Interface, Comunicação, Saúde, Educação; Estudos Avançados; Psicologia USP. Assim, pode-se perceber que há maior produção nas áreas de enfermagem e psicologia, sendo estas áreas integradas ou independentes, bem como evidenciou-se maior interesse do tema violência e vulnerabilidade na perspectiva da saúde coletiva.

$\mathrm{Na}$ análise dos artigos observaram-se cinco estudos envolvendo crianças e adolescentes, o que reflete uma preocupação dos estudiosos com essa parcela da população. A ideia de que o lar é o local de maior segurança e que confere proteção à criança nem sempre é correta, pois a maioria dos casos de maus tratos na infância ocorre no ambiente familiar e permanece, em grande parte, silenciosa, inacessível a observações superficiais não dirigidas para 0 problema. ${ }^{5}$ Nessa perspectiva, um dos estudos aponta a violência física sendo empregada pelos pais como prática disciplinar. $^{10}$

Os demais artigos referem-se a populações vulneráveis como: povos indígenas, gênero feminino, portadores de HIV, trabalhadores informais de rua, povos assentados e populações pobres, dependentes de serviços públicos. Com isso constata-se uma situação atual de nossa sociedade, em que se tem nestas populações a parcela mais vulnerável a atos e situações de violência, seja ela, direta ou indireta, de cunho físico ou psicológico.

Assim, com suporte nos conteúdos destes quinze 
artigos, emergiram duas categorias: estado e contexto social como gerador da violência e vulnerabilidade; implicação da violência e vulnerabilidade nos serviços de saúde, as quais serão apresentadas e discutidas na sequência.

\section{ESTADO E CONTEXTO SOCIAL GERADORES DA VIOLÊNCIA E VULNERABILIDADE}

Os artigos analisados apresentaram dados que identificam o Estado como gerador de violência e vulnerabilidade quando apontam para uma ação do poder público, caracterizada pela opressão física e psíquica na tentativa de manter a ordem. Nessa perspectiva, as pesquisas revelam que os cidadãos, por encontrarem-se em condições sociais desfavoráveis de insegurança, buscam na ilegalidade uma forma de sobrevivência. ${ }^{6-9}$

Ainda, outros estudos apontam a vulnerabilidade social como causa de violência, considerando o contexto social onde estão inseridos os sujeitos das pesquisas como determinante da vulnerabilidade. . $^{10-14}$ Ainda é revelado em um dos estudos, que o gênero feminino e a raça negra não são fatores determinantes, mas favorecem o desencadeamento do ato. ${ }^{15}$

Em estudo que abordou os significados de morte entre crianças pré-escolares, seus pais e professores, foram encontrados a banalização e a naturalização como justificativa para a perpetuação da violência. ${ }^{12}$

\section{IMPLICAÇÕES DA VIOLÊNCIA E VULNERABILIDADE PARA OS SERVIÇOS DE SAÚDE}

Nessa categoria, os estudos evidenciaram a necessidade dos serviços de saúde aderirem à problemática da violência e vulnerabilidade como forma de contribuir para a prevenção e proteção deste agravo, já que a violência, em suas diferentes formas e manifestações, tem ampla repercussão para os indivíduos, suas famílias e para a sociedade, tanto que vem sendo considerada como um problema de saúde pública. Uma das maneiras de intervenção dos serviços de saúde em prol da diminuição dos eventos violentos e da vulnerabilidade aponta para a intersetorialidade, as redes de apoio, a interdisciplinaridade, o aprimoramento do sistema de informação, a formação e educação permanente dos profissionais da saúde. 9,10,15,
$17-21$

Quanto à intersetorialidade e as redes de apoio, as reflexões trazidas pelas publicações indicam a necessidade de construir vínculos e aproximações por meio de atividades de convivência e atendimento integral com práticas intersetoriais. Dentre essas se destaca a oferta de atividades culturais, esportivas, terapêuticas e parcerias entre instituições públicas e privadas.

Um desafio apontado por dois estudos, ${ }^{15,18} \mathrm{diz}$ respeito à união entre várias áreas de trabalho como saúde, educação e cultura almejando que se entrecruzem e se conectem somando esforços para a mudança de rotas de vidas em situação de violência e vulnerabilidade social.

Outra questão apontada por um dos artigos, ${ }^{17}$ que implica diretamente nos serviços de saúde, trata-se do sistema de informação da morbimortalidade por causas externas em nosso país. Embora se reconheça que as mortes por violências e acidentes sejam mais bem informadas do que as naturais há ainda muito a ser feito enquanto os legistas não alcançarem um patamar ideal no preenchimento das declarações de óbitos. ${ }^{22}$

Outro dado relevante, observado em uma pesquisa ${ }^{17}$ é que mulheres são vítimas de acidentes domésticos relacionados com as atividades que desenvolvem na família e na vida social, indicando que a violência doméstica mantém-se velada ou subnotificada. Esses estudos evidenciam que o sistema necessita do aprimoramento dessas informações, a fim de subsidiar políticas públicas de prevenção e melhoria no atendimento às vítimas de violência. ${ }^{15,17}$ Os autores desses estudos sugerem a adoção de registro hospitalar detalhado, que identifique os tipos de contextos sociais em que ocorrem os acidentes e violências, bem como o desenvolvimento de formulários de registro que impeçam o ocultamento de dados.

A necessidade de formação e capacitação adequada dos profissionais de saúde a respeito desta temática, também foi apontada por alguns estudos. 7,8,19 A intenção é que os profissionais envolvidos efetivamente se engajem no contexto no qual pretendem intervir para que possam contribuir com a prevenção e até mesmo a reabilitação dos sujeitos que sofrem de violência ou que se encontram em situação 
de vulnerabilidade. Destaca-se ainda, em um dos estudos. $^{10}$ a importância do papel do enfermeiro inserido nessa temática.

\section{DISCUSSÃO}

Frente aos resultados acima apresentados é possível inferir que a categoria referente ao Estado e contexto social como geradores da violência e vulnerabilidade encontra respaldo em duas teorias. Uma delas explica as raízes da violência como um fenômeno resultante dos acelerados processos de mudança social. Tal fenômeno é explicado pela industrialização, pois provocou fortes correntes migratórias para grandes centros urbanos, levando a população a viver sob condições de extrema pobreza, desorganização social, expostas a novos comportamentos e sem condições econômicas de realizarem seus anseios. $^{23}$

A outra teoria explica a violência e o crescimento dos índices de criminalidade no país pela falta de autoridade do Estado, sendo que esta é caracterizada por ser repressiva. Veicula-se a crença num Estado neutro, árbitro dos conflitos e mantenedor da ordem em benefício de todos, à margem da questão das classes, dos interesses econômicos e políticos. ${ }^{23}$ Essas ideias combinam com o senso comum, que é a favor da força repressiva como condição de ordem e progresso, assim como pôde-se observar em um estudo ${ }^{24}$, em que a concepção de violência dos sujeitos da pesquisa referiase a força repressiva do Estado como forma de diminuir ou sanar os problemas de delinquência dos indivíduos.

A violência ainda pode ser explicada pela vulnerabilidade social caracterizada pelo baixo grau de escolaridade, dificuldades de acesso à educação, trabalho, saúde, lazer, e cultura; rupturas com laços familiares, a disputa pelo domínio do tráfico de drogas e violência familiar presenciada no seu núcleo. Sendo assim, enfatizam-se nos estudos sobre violência causada pela vulnerabilidade social, duas de suas representações: a estrutural e a familiar. ${ }^{9}$

Com referência a violência estrutural apontam-se as questões de preconceito racial e de gênero, na qual a conduta política do estado e de seus governantes podem privilegiar alguns grupos em detrimento de outros, influenciando nas desigualdades e contribuindo para a exclusão. ${ }^{9}$ Destaca-se, assim, a violência estrutural conceituada como sinônimo de injustiça social. ${ }^{24} \mathrm{Com}$ isso, entende-se que as políticas governamentais do Estado são ambíguas, de natureza múltipla e complexa no estabelecimento de metas e também na execução, deixando espaço para que ocorram conflitos e exclusões, que acabam por atingir a sociedade direta ou indiretamente. ${ }^{9}$

Portanto, o Estado e suas políticas governamentais são vistos como geradores de insegurança, pois favorecem que ocorram condições de exclusão e esquecimento, tornando a sociedade mais vulnerável à violência. Nesse sentido, tais desigualdades tornam turbulentas as relações entre as diversas camadas socioeconômicas validando alguns aspectos da vulnerabilidade. ${ }^{9}$

Nesse contexto, destaca-se como um dos achados dessa pesquisa a banalização e naturalização da violência, que se acredita ocorrer, também, em virtude da exposição intensa da sociedade aos atos violentos e a mídia, o que pode gerar uma certa confusão em relação a estes aspectos. Porém, acredita-se que para romper com esse conceito, a violência não deve ser vista como um fato natural, mas influenciada por elementos culturais e sociais, a fim de poder ter uma compreensão mais abrangente das suas causas. ${ }^{23}$

Quanto à categoria implicações da violência e da vulnerabilidade para os serviços de saúde, acredita-se que há a necessidade de ações públicas integradas com as diversas disciplinas do conhecimento humano e a multiprofissionalidade ${ }^{23}$ como um meio de prevenção e proteção da violência na área da saúde. Essa é uma exigência epistemológica intrínseca e essencial na qual, o princípio da cooperação, deve prevalecer sobre a hierarquia das disciplinas. Ainda, pode-se dizer que o contrário de violência não é a não-violência e sim a cidadania e a valorização da vida humana. ${ }^{23}$

Tratando-se da questão do sistema de informação da morbimortalidade, a discussão deve permear a gestão e a atenção em saúde, já que os serviços de saúde se deparam com as condições e informações insuficientes quanto às notificações por violência o que dificulta a viabilização de ações preventivas nos diversos setores. Para tanto, a formação do profissional de saúde quanto aos aspectos inerentes à violência e vulnerabilidade, reitera-se tanto a 
importância da Política Nacional de Educação Permanente em Saúde (PNEPS) ${ }^{25}$ de 2006, como as Diretrizes Curriculares Nacionais dos Cursos de Graduação em Saúde de 2001. ${ }^{26}$ A PNEPS visa, entre outros, contribuir para transformar e qualificar as práticas de saúde e a organização das ações e dos serviços. As Diretrizes indicam a necessidade dos cursos distanciarem-se da índole tecnicista, alheia ao contexto social e descomprometida com as reais necessidades de saúde da população e aproximarem-se de uma visão crítico-reflexiva, na qual a formação profissional esteja engajada com as mudanças que se fazem imprescindíveis na saúde. Assim, com relação a este novo perfil dos profissionais da área da saúde, academicamente formados para pensar e agir criticamente perante a problemática social, pode-se pensar em mudanças positivas do contexto atual de vulnerabilidades e violência.

\section{CONSIDERAÇÕES FINAIS}

Os resultados encontrados permitem afirmar que os artigos publicados no período de dez anos, em comparação à produção com a temática do estudo, são bastante escassos diante da relevância e das implicações sociais, na saúde, causadas pela violência e vulnerabilidade. Nesse estudo, identificou-se o Brasil como o país com mais publicações a esse respeito e São Paulo como o estado brasileiro com maior produção. Constatou-se a preferência pela pesquisa quantitativa, que tem por objetivo elucidar dados, indicadores e tendências. Quanto aos sujeitos dos estudos, as crianças e os adolescentes representaram a maior preocupação dos estudiosos. A área de estudo que contemplou maior número de publicações foi a Enfermagem, seguida da Psicologia e Antropologia. Diante do quadro, percebeuse a preocupação do enfermeiro para com a temática, $o$ que contribui para implantação de medidas efetivas de intervenção nos aspectos de vulnerabilidade social, e por consequência no combate à violência.

Os artigos analisados identificaram o Estado e o contexto social como geradores de violência e vulnerabilidade. O poder público tenta manter a ordem por meio da opressão física e psíquica ou adota postura inerte perante a realidade dos sujeitos. Por sua vez, a sociedade inserida no contexto de injustiça social, provoca a banalização da violência e colabora para sua perpetuação. Para romper com esse conceito da violência e, por conseguinte, diminuir os eventos violentos e a vulnerabilidade, os estudos sugerem que a área da saúde adote ações intersetoriais e interdisciplinares, o aprimoramento do sistema de informação e a formação e educação permanente dos profissionais da saúde.

Diante do exposto, percebe-se uma concepção ampliada da temática violência e vulnerabilidade nas publicações analisadas, o que vai ao encontro dos pressupostos do Sistema Único de Saúde (SUS) e, por conseguinte, das Diretrizes Curriculares Nacionais para os Cursos de Graduação em Saúde. O SUS toma a saúde como direito universal a todo o cidadão garantido mediante políticas públicas. Nesse sentido, o sistema de saúde do país não se restringe à prestação de serviços assistências, mas por incorporar a participação social e a intersetorialidade como diretriz e princípio, estimula movimentos e ações contra situações geradoras de violência e vulnerabilidade. As Diretrizes Curriculares Nacionais dos Cursos de Graduação em Saúde constituem orientações às instituições formadoras de recursos humanos na área da saúde tendo como referência o SUS. Para tanto, implica em formar profissionais com competência para analisar os problemas da sociedade, que seja responsável e comprometido com a resolução de problemas e assuma papel de ator social nas mudanças que se tornam necessárias para superar as precárias condições de saúde em que vive a população brasileira, bem como para participar na construção ou ampliação dos direitos de cidadania.

Por último, em termos de lacunas, pode-se destacar que pesquisas ainda se fazem necessárias para que sejam aprofundadas as questões de violência e vulnerabilidade. Nesse sentido, as investigações com abordagem qualitativa podem trazer contribuições para que a temática seja compreendida na perspectiva subjetiva e sociocultural.

\section{REFERÊNCIAS}

1.Minayo MCS. A difícil e lenta entrada da violência na agenda do setor saúde. Cad. Saúde Pública 2004 Mai-Jun 20(3):646-7.

2. Brasil. Ministério da Saúde. Análise dos Dados de mortalidade no Brasil e regiões, 2002. Boletim do Sistema 
de Informações sobre Mortalidade. Brasília: Ministério da Saúde, DF, 2002. [online] [acesso em 04 de julho de 2009]. Disponível em: http: www.saúde.gov.br/vigilanciaepidemiologica.

3. Ayres J RCM, França Júnior I, Calazans GJ, Saletti Filho HC. Conceito de vulnerabilidade e as práticas de saúde: novas perspectivas e desafios. In: Czeresnia D, Freitas CM (org), Promoção da Saúde. Rio de Janeiro: Fiocruz; 2003.p.117-139.

4. Minayo MCS. Contradições e consensos na combinação de métodos quantitativos e qualitativos. In: Minayo MCS. $O$ desafio do conhecimento. São Paulo: Hucitec; 2008. p.5576.

5. Cavalcanti AL. Maus-tratos infantis: aspectos históricos, diagnóstico conduta. Pediatria Moderna. 2002 Set; 38(9): 421-6.

6. Itikawa L. Vulnerabilidade do trabalho informal de rua: violência, corrupção e clientelismo. São Paulo em Perspectiva 2006 Jan-Mar 20(1): 136-147.

7. Alvarez LE. De jóvenes, actos delectivos y responsabilidades. Estudos e Pesquisas em psicologia. 2005; 5(2):96-106.

8. Lopes RE, Adorno RCF, Malfitano APS, Takeiti BA, Silva CR, Borba PLO. Juventude pobre, violência e cidadania. Saúde e Sociedade. 2008 p. 63-75.

9. Scott P. Re-assentamento, saúde e insegurança em Itaparica: um modelo de vulnerabilidade em projetos de desenvolvimento. Saúde e sociedade. 2006 Set-Dez; 15(3):74-89.

10. Carmo CJ, Harada MJCS. Physical violence as educational practice. Rev. Latino-Am. Enfermagem 2006; 14(6): 849-856.

11. Poletto M, Koller SH, Dell'Aglio DD. Eventos estressores em crianças e adolescentes em situação de vulnerabilidade social de Porto Alegre. Ciências \& Saúde Coletiva. 2009 14(2):455-466.

12. Jucá VJS, Silva ACN, Passos CM, Castro GA, Melo GB, Tortorella I et al. Significando a morte, através de redes sociais, em um contexto de vulnerabilidade social -um estudo com crianças pré-escolares, seus pais e professores. Psicologia \& Sociedade 2007 19(2):122-130.

13. Zaluar A. Democratização inacabada: fracasso da segurança pública. Estudos Avançados 2007 Set-Dez 21(61): 31-49.
14. Coimbra JR, Alvares CE, Santos, RV. Saúde, minorias e desigualdade: algumas teias de inter-relações, com ênfase nos povos indígenas no Brasil. Ciênc. saúde coletiva. 2000, 5 (1):125-132.

15. Diniz NMF, Almeida LCG, Ribeiro BCS, Macêdo VG. Women victims of sexual violence: adherence to chemoprevention of HIV1.Rev. Latino-americana de Enfermagem 2007 Jan-Fev 15(1):7-12.

16. Meneghel SN, Barbiani R, Steffen H, Wunder A P, Dalla Roza M, Rotermund J, Brito S, Korndorfer C . Impacto de grupos de mulheres em situação de vulnerabilidade de gênero. Cad. Saúde Pública. 2003,19 (4): 955-963.

17. Santos JLG, Garlet ER, Figueira RB, Lima SBS, Prochnow AG. Acidentes e violências: caracterização dos atendimentos no pronto-socorro de um hospital universitário. Saúde Sociedade 2008 17(3):211-218.

18. Lopes RE, Malfitano APS. Ação social e intersetorialidade: relato de uma experiência na interface entre saúde, educação e cultura. Interface. 2006 Jul-Dez 10(20): 505-15.

19. Silva GF, Silva MDB, Silva LR, Santos IMM. Violência contra mulher gestante sob a visão de puérperas. Rev Enferm UFPE On Line. 2009 jul/set;3(3):33-42.

20. Paiva V, Peres C, Blessa C. Jovens e adolescentes em tempos de Aids reflexões sobre uma década de trabalho de prevenção. Psicol. USP. 2002,13 (1): 55-78.

21. Arevalo, L A P; Klijn, T. P. Percepcion de violencia y factores asociados segun usuarios de un consultorio $y$ postas de salud. Concepcion, chile. Cienc. enferm. [online]. 2006, 12 (1): 39-51.

22. Jorge MHPM, Gotlieb SLD, Laurenti R. O sistema de informações sobre mortalidade: problemas e propostas para o seu enfrentamento II - Mortes por causas externas. Rev. Bras. Epidemiol 2002 5(2):212- 223.

23. Minayo MCS, Souza ER. Violência e saúde como um campo interdisciplinar e de ação coletiva. História, Ciência, Saúde.1997 Nov-Fev 4(3): 513-31.

24. Budó MLD, Mattioni FC, Machado TS, Ressel LB, Borges ZN. Concepções de violência e práticas de cuidado dos usuários da estratégia de saúde da família: uma perspectiva cultural. Texto e Contexto 2007. Jul-Set; 16(3): 511-9. 
25. Brasil. Ministério da Saúde. Secretaria de Gestão do Trabalho e da Educação na Saúde. Departamento de Gestão da Educação em Saúde. Política Nacional de Educação Permanente em Saúde. Brasília, 2009. 64 p. Disponível em:

http://portal.saude.gov.br/portal/arquivos/pdf/volume9.pdf. Acesso em: 21 de outubro, 2010.

26. Brasil. Ministério da Educação. Conselho Nacional de Educação. Diretrizes Curriculares Nacionais dos Cursos de Graduação em Enfermagem, Medicina e Nutrição. 2010, p. 38. Disponível em: http://portal.mec.gov.br/dmdocuments/ces1133.pdf

\section{Correspondência}

Maria de Lourdes Denardin Budó

Rua Appel, 800/208. Bairro Fátima. 97015 - 030 - Santa Maria - RS

E-mail: lourdesdenardin@gmail.com

Recebido em 02 de setembro de 2010.

Aprovado em 30 de novembro de 2010. 